Relations industrielles

Industrial Relations

\title{
Insécurité d'emploi et relations de travail dans l'industrie canadienne de la construction, par Paul Malles, Ottawa, Conseil économique du Canada, 1975, 122 pp.
}

\section{Jean Bernard Fournier}

Volume 31, numéro 1, 1976

URI : https://id.erudit.org/iderudit/028697ar

DOI : https://doi.org/10.7202/028697ar

Aller au sommaire du numéro

Éditeur(s)

Département des relations industrielles de l'Université Laval

ISSN

0034-379X (imprimé)

1703-8138 (numérique)

Découvrir la revue

Citer ce compte rendu

Fournier, J. (1976). Compte rendu de [Insécurité d'emploi et relations de travail dans l'industrie canadienne de la construction, par Paul Malles, Ottawa, Conseil économique du Canada, 1975, 122 pp.] Relations industrielles / Industrial Relations, 31(1), 161-162. https://doi.org/10.7202/028697ar

Tous droits réservés (C Département des relations industrielles de l'Université Laval, 1976
Ce document est protégé par la loi sur le droit d'auteur. L'utilisation des services d'Érudit (y compris la reproduction) est assujettie à sa politique d'utilisation que vous pouvez consulter en ligne.

https://apropos.erudit.org/fr/usagers/politique-dutilisation/ 
de structurer des programmes ou services de main-d'œuvre. Le Québec aurait présentement grand intérêt à suivre l'exemple du Manitoba. En second lieu, un tel exercice indique clairement l'orientation que prendra la politique de main-d'œuvre dans cette province: corriger le marché du travail. Même si cette leçon peut sembler évidente à plusieurs, elle est d'autant plus utile qu'elle sert à identifier clairement la mission et le champ d'action propre à une telle politique publique et peut servir à limiter les problèmes de juridiction entre ministères de même juridiction dans le domaine de la main-d'œuvre.

Le comité fait au total huit recommandations très générales qui frisent souvent le vœu pieux et qui touchent plusieurs aspects d'une politique de main-d'œuvre (P.M.O.). Cependant ces huit recommandations montrent clairement que le E.D.A.B. n'a pas encore une idée claire et bien structurée de ce qu'est une véritable P.M.O. du moins théoriquement. On a aboutit alors à identifier la principale faiblesse de ce rapport: l'absence d'une conception claire et d'un cadre de référence structuré sur la P.M.O.

Quant à la seconde partie de ce rapport, on y retrouve neuf études portant sur certains aspects économiques très particuliers du marché du travail. Ces études, généralement bien faites, représentent un effort d'exploration utile à l'élaboration d'une P.M.O. Cependant, un tel effort devra se poursuivre pour en arriver à définir une P.M.O. pour le Manitoba.

En résumé, cet ouvrage est intéressant à plusieurs égards. Il montre l'effort d'une province à se définir une P.M.O. propre à ses besoins. Cet effort, qui s'inscrit dans le mouvement de provincialisation de la P.M.O. au Canada, n'est cependant pas complet. Un peut neanmoins retirer des leçons utiles autant des aspects forts que des carences de ce rapport.

Jean SEXTON

Université Laval

Insécurité d'emploi et relations de travail dans l'industrie canadienne de la construction, par Paul Malles, Ottawa, Conseil économique du Canada, 1975, $122 \mathrm{pp}$.

Cet ouvrage de Paul Malles constitue un document préparatoire au rapport du Conseil économique du Canada, rapport portant sur l'instabilité dans l'industrie de la construction et intitulé: "Pour une croissance plus stable de la construction».
Cette étude se penche plus particulièrement sur l'effet de l'instabilité sur les relations du travail dans l'industrie canadienne de la construction.

L'industrie de la construction est très différente des autres industries. À preuve, bien que n'employant que $6 \%$ de la maind'œuvre, elle compte pour $19 \%$ des jourshomme perdus. Dans le chapitre consacré aux facteurs d'instabilité, l'auteur insiste sur l'importance des effets secondaires des conflits du travail à l'intérieur de l'industrie de la construction. Cette importance des effets secondaires est une autre différence entre l'industrie de la construction et les autres. Un autre facteur d'instabilité est celui de la disparité salariale existant entre le travailleur de l'industrie de la construction et celui des autres industries.

Un aspect qui ressort tout au long d l'ouvrage, est celui du statut particulier que détient le Québec en regard des autres provinces. Ces différences seront perçues aussi bien du point de vue juridique (bill 290 , décret de la construction, loi 9) que syndical (rivalité syndicale).

L'auteur parle des syndicats ouvriers de la construction. Il est intéressant de souligner le pouvoir conservé par les sections locales dans les syndicats internationaux de la construction ainsi que le peu de rapport entre celles-ci. Ce n'est que dernièrement qu'est apparu le Comité consultatif pour les métiers de la construction au Canada. Les associations patronales ont l'originalité d'être beaucoup plus organisées que celles des autres industries avec l'Association canadienne de la construction (ACC) et ajoutons, au Québec, avec la toute nouvelle Association des entrepreneurs en construction du Québec.

Un chapitre entier est consacré à la législation concernant les relations du travail. L'auteur présente sommairement les différentes législations dans le domaine de la construction et ce pour chaque province du Canada. Dans ce même chapitre, une attention particulière est portée au «système de décrets » de la province de Québec.

L'auteur présente également tout au long d'un chapitre les avantages et les inconvénients de la centralisation des relations du travail à laquelle il attribue comme inconvénients majeurs la multiplication des conflits et la présence de grèves illégales ou «sauvages».

Le type de conventions collectives de la construction est analysé dans un chapitre de cet ouvrage. On y constate par exemple la tendance pour la province d'Ontario à 
favoriser le type de convention provinciale par métier. L'auteur s'appuie sur l'étude de 485 conventions collectives types de tout le Canada sauf du Québec (à cause de son «système de décrets»). Il constate que l'atelier fermé est favorisé dans l'industrie de la construction. Les expressions «sécurité syndicale» et «sécurité d'emploi» sont devenues synonymes dans cette industrie alors que dans les autres industries, il n'existe pas de relation directe de cause à effet entre la «sécurité d'emploi» et la "sécurité syndicale». Ici, d'après l'auteur ces expressions sont synonymes parce que la protection de l'emploi a constitué l'objectif principal, la sécurité syndicale ne venant qu'en deuxième lieu.

On a soutenu que les bureaux de placement syndicaux constituaient une abdication de la fonction patronale et qu'ils contribuaient à créer des pénuries artificielles de main-d'œuvre. L'auteur justifie cependant leur présence par la nature même du rapport employeur-travailleur dans cette industrie et la courte période d'emploi chez un même employeur. L'inconvénient majeur de ces bureaux, selon l'auteur, n'est pas tant l'abus de pouvoir ou la discrimination qu'ils peuvent occasionner mais plutôt les difficultés qu'ils entraînent dans l'établissement d'une politique de maind'œuvre d'envergure nationale. L'auteur suggère la création de centres spéciaux de main-d'œuvre de la construction sous une administration tripartite en guise de remplacement des bureaux actuels.

Dans le chapitre réservé aux problèmes de juridiction, l'auteur indique deux types principaux de différends: querelles de reconnaissance syndicale et conflit d'attribution de tâches. Ici encore, le Québec est un cas particulier puisqu'on $y$ retrouve de nombreuses querelles de reconnaissance syndicale contrairement aux autres provinces. Au Québec, ces querelles atteignent leur point culminant lors de la période de «maraudage". Les facteurs faisant varier l'impact des conflits d'attribution sont l'instabilité d'emploi et le changement technologique.

Un chapitre entier traite de la protection de la main-d'œuvre soit par l'application de normes de travail et de sécurité, soit par les régimes de retraite. Au sujet de l'application des normes de travail et de sécurité, l'auteur insiste sur le lien existant entre l'instabilité d'emploi, l'inconstance des relations du travail et le taux élevé d'accidents survenant dans l'industrie de la construction. Notons que le Québec est la seule province à avoir un système d'avantages sociaux organisé à l'échelle provinciale.
Dans son dernier chapitre, l'auteur insiste sur l'importance que prend l'industrie de la construction par des chantiers éloignée dans le Nord canadien. Il parle plus précisément des problèmes qui apparaissent à la suite de ce nouvel essor.

Dans cet ouvrage, l'auteur a su répondre à l'objectif qu'il s'était fixé à savoir: étudier l'insécurité d'emploi et les relations du travail dans l'industrie canadienne de la construction. L'étude qui a été faite permet au lecteur de mieux comprendre ce monde particulier dans le domaine des relations professionnelles qu'est l'industrie de la construction. On doit cependant regretter tout au long de l'ouvrage que la traduction française laisse à désirer. Ainsi, on remarque: relations de travail pour relations du travail, déstabilisation pour instabilité etc. Souvent pour bien comprendre, on est obligé de se reférer à la version anglaise.

\section{Jean Bernard FOURNIER}

Université Laval

Labor in the United States, by Sanford Coher, Ontario, Charles E. Merrill Publishing Division, Bell \& Howell Canada Ltd., 1975, 479 pp.

The purpose of this book is to familiarize the reader with Labour Issues in the U.S.A. In the preface the author says that he has resolved the problems of what to include and to exclude by maintaining «an appropriate blend of back-ground considerations and contemporary development». Has he really succeeded in this respect? A detailed review of the material in this book reveals an over emphasis on the historical forces and back-ground knowledge on one hand and a very short and superficial treatment of the contemporary issues on the other.

The book deals with six major areas: 1) The Labor Force; 2) Organized Labour; 3) Collective Bargaining; 4) Wage Analysis 5) Labour Law; 6) Unemployment Problems and Programs.

The book deals with six major areas: 1) The Labor Force; 2) Organized Labour; 3) Collective Bargaining; 4) Wage Analysis 5) Labour Law; 6) Unemployment Problems and Programs.

In the first part the author provides elaborate historical statistical data of the labor force in the U.S.A., reflecting the natural rate of population growth, the volume of immigration and shifts in distribution of the non agricultural labor force 